\title{
The Impact of Coherent Light Sources On Luminance Measurement
}

\author{
Gon Kim, Hong Soo Lim, and Jeong Tai Kim
}

\begin{abstract}
The algorithms and devices used in measuring luminance have a significant effect on the results to be obtained. The two available methods of measuring luminance have shown that luminance of two identical lighting sources can be totally different owing to certain other factors. Consequently, the current paper examined the effect that distance and a coherent light source may have on a target light source in regards to measured luminance. The results indicate that when the distance from the light source is increased on the outdoor, there is a decrease in measured luminance. For the second objective, the results showed that measured luminance increases by about $3 \%$ and $2 \%$ when the coherent light source is on and oriented towards the point of measurement at $15^{\circ}$ and $30^{\circ}$ respectively.
\end{abstract}

Index Terms - Luminance, HDR image processing, coherent light source.

\section{INTRODUCTION}

Luminance measurements can be obtained through surface luminance measurement using HDR (High Dynamic Range) image processing and point luminance measurement using CS-100 luminance meter devices. The quantity of light received at the device is converted into luminance. Normally, Luminance can be classified into luminance that is directly from the light source and luminance that is reflected from object or surfaces such as walls, floors, ceilings and the sorts [1]. Thus, the measured luminance values differ according to the distance from a light source and light source's orientation, and such should be considered before proposing the criteria forassessing light pollution. Furthermore, when measuring an outdoor light source, according to the luminance measurement method, any light source in the surrounding area may have an influence on the luminance of the targeted light source.

Recently, the Korean government shifted their attention on the effect that light pollution has on visual environment, high energy consumption, view of the night time sky as well as its adverse effects on well-being of plants and animals.Consequently, Korean standards for the purposes of reducing and preventing were put in place. These are to be followed when installingroad lights, lights for residential buildings, commercial advertisements, sports facilities, parks and so forth.

There are several research projects aimed at developing methods to be used in evaluating luminance and illuminance displayed by existing light sources. Also, many researchers

Manuscript received June 1, 2014; revised July 23, 2014. This work was supported by the National Research Foundation of Korea (NRF) Grant funded by the Korea government (MSIP) (No. 2008-0061908).

The authors are with the Architectural Engineering Department, Kyung Hee University, Yongin, Rep. of Korea (e-mail: gonkim@khu.ac.kr, hongsoolim@khu.ac.kr, jtkim@khu.ac.kr). are focusing on studying the appropriateness and accuracy of the already existing techniques used to measure different quantities of light such as luminance and illuminance. Therefore, this study examines luminance changes according to the alteration of distance and the relation between the measured light source and its surroundings.

\section{LuminanCe MEASUREMENT THEORIES}

\section{A. Algorithm of Luminance}

Luminance can be defined as the intensity of light perceived by a human eye. In display and entertainment industries, luminance refers to the intensity of light at which images are displayed.

In buildings, luminance can be definedas the brightness of light radiated from light fixtures or reflected from building surfaces or objects within the building that reaches the human eye. Severe luminance difference by indoor building surfaces are likely to cause imbalance in brightness within a particular area. In addition, diverse building surfaces and objects possessing very high differences in their respective luminance levels lead to visual discomforts or in few cases visual impairment. In order to reduce issues related to visibility therefore, designers try to minimize luminance differences caused by different finishing materials, artificial lighting fixtures, daylighting, irregular space designs and so forth. The CIE (Commission Internationale de l'Eclairage) gives the formula below to be used generally for luminance estimations.

$$
L=\frac{d^{2} \Phi}{d A d \Omega \cos \theta}
$$

$L$ is the luminance $(\mathrm{cd} / \mathrm{m} 2)$

$\Phi$ is the luminous flux or luminous power ( $\mathrm{lm}$ ),

$\Theta$ is the angle between the surface normal and the specified direction,

$A$ is the area of the surface (m2), and

$\Omega$ is the solid angle (sr).

The equation shown above gives the value of luminance $(L)$ that is being radiated directly from the light source. However, when dealing with buildings and other enclosed spaces, the luminance reaching an eye after the light has been reflected several times on surfaces and objects should be considered as it also plays a significant role in regards to visibility.

$$
L_{v}=\frac{d^{2} \Phi_{v}}{d A d \Omega \cos \theta}
$$

$L$ is the 'visual' luminance $\left(\mathrm{cd} / \mathrm{m}^{2}\right)$ 
$\Phi$ is the 'visual' luminous flux or luminous power ( $\mathrm{lm})$,

$\Theta$ is the angle between the normal of surface and the specified direction,

$A$ is the area of the surfac $\left(\mathrm{m}^{2}\right)$, and

$\Omega$ is the solid angle (sr).

Unlike formula (1), formula (2), considers the estimated luminance from a human eye perspective. Whilst, the obtained luminance is derived from the same quantity as that of formula (1), visibility and luminance are both merged in the final estimate. The same formula can be utilized for both point and surface luminance measurement techniques. These two techniques are not based on estimating absolute luminance values of illuminating surfaces but instead consider that luminance levels are subjected to change depending on the velocity of the light [2].

\section{B. Measuring Devices of Luminance}

There are two methods adopted for measuring luminance namely point luminance measured by using either CS or LS equipment and surface luminance measured by using HDR image processing techniques. CS and LS equipment have been noted for their inaccuracy when measuring luminance on light source surfaces. For example, when using LS 100 for measurements and the measuring angle exceeds $1^{\circ}$, the errors in the measured luminance can be greatly reduced if the diameter of the surface whose luminance is being measured is set to at least $14.4 \mathrm{~mm}$ and the minimum distance from the surface to the measuring point set at a minimum distance $0 \mathrm{f}$ $1014 \mathrm{~mm}$ (40 inches) [3]. This indicates that measured luminanceis greatly affected by the diameter of the surface whose luminance is being measured as well as the distance between the point of measurement and the surface being measured. Fig. 1 shows the CS-100 luminance meter device utilized for measuring point luminance.

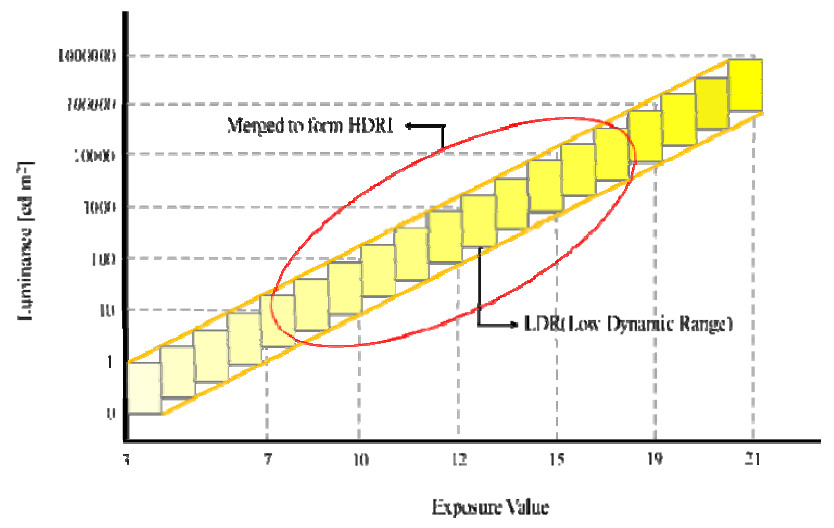

Fig. 1. Correlation of exposure value and luminance.

In the case of CS-100 and LS-100 optical instruments, SLR (Single-Lens-Reflex) optical meter is used for measurements. This generates a sufficient level of accuracy at the same time high-brightness to be also measurement. However, in case of HDR, it uses the image sensor of CCD, not the photoelectric element measured using a digital camera, in order to obtain luminance information as the collection of RGB digital information per pixel [4]. HDR imaging techniques range back to a few decades ago. It is being applied in camera designs, software image processing, movie productions and so forth. HDRI is quickly replacing conventional photography. The main reason for this is that HDRI centers on the relationship between pixels rather than their individual values to control image appearance [5]. A single image shot using a digital camera can have a maximum luminance contrast of 1: 10000, such luminance conditions cannot be detected by a human eye. Therefore High Dynamic range (HDR) technology is used to merge so many low contrast images and thus increasing their luminance contrast to about 1: 100000 which can then be detected by a human eye. Fig. 1 shows the relation between exposure value and measured luminance [6].

HDR image processing can also be used to measure luminance values of uneven surfaces, however color temperature, size of the light source and the distribution of the light may give rise to variations in measured luminance [3]. Such factors should be considered when implementing "the act on prevention of light pollution caused by artificial lighting"

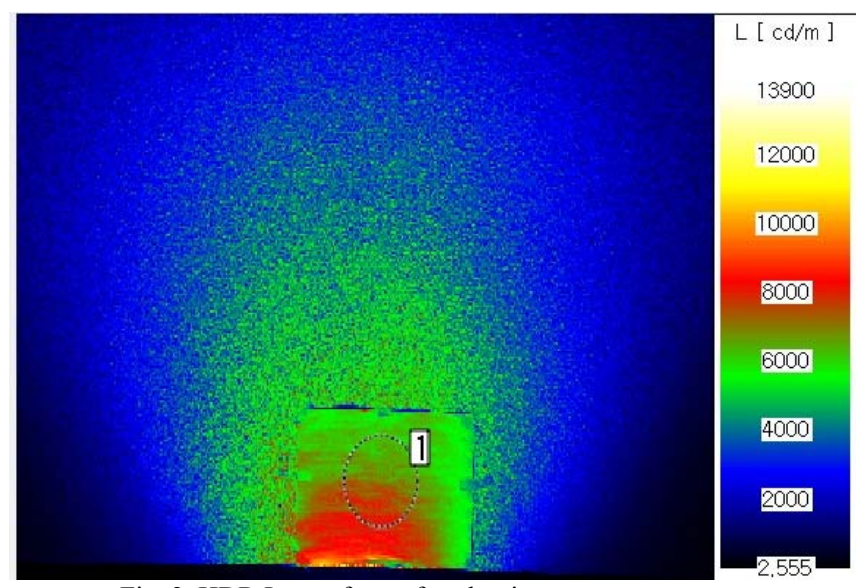

Fig. 2. HDR Image for surface luminance measurement.

Fig. 2 shows a sky simulator located in Kyung Hee University, South Korea, where luminance measurements using HDRImagingwas performed. A sky simulator is an equipment whose main purpose is to emulate the appearance of an ordinary sky. The circle numbered 1 in the picture above shows the range of luminance estimation. Also, the colorful regions shown in the picture indicate the distribution of the evaluated luminance.

\section{Methodology: Luminance Interference Caused by a Neighboring Light Source}

The present paper discusses the effect of a coherent light source on the luminance displayed by a target source. Past theoretical studies considered luminance changes in regards to diffraction, chromatic and spherical aberrations of the eye [7], [8] but very few studies have dealt with the impact of lighting physical characteristics such as distance from light source, reflectance and angle on the perceived brightness [9]. Also, research studies have used HDR photography as a visual assessment tool as well as to collect luminance and luminance contrast data to be used in the analysis of human's visual field [10]. In the current study therefore, we used CS-100 to measure the luminance displayed by the target area when the coherent light source is illuminating and pointed towards the CS-100 device at different angles. One extra light source was installed in the sky simulator to serve as a neighboring light source (Fig. 3) 
The distance from the target surface to the point of measurement is 3 meters, and the angles of coherent light source were changed from 0 degree to 30 degrees. In order to minimize errors in the experiment, CS-100 luminance meter only was used to measure the luminaire influence. The target light source and coherent light source are of a similar nature. In addition, each surface was measured 3 times during the experiment, and the average of the results presented.

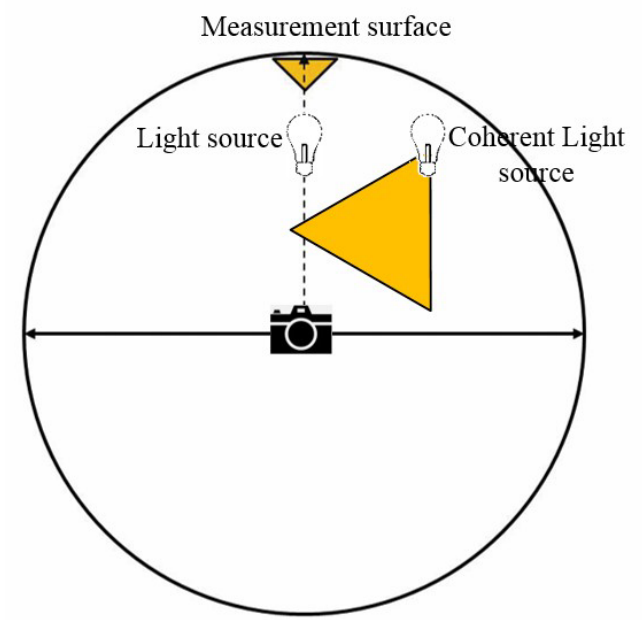

Fig. 3. Evaluation of coherent luminaire influence on luminance.

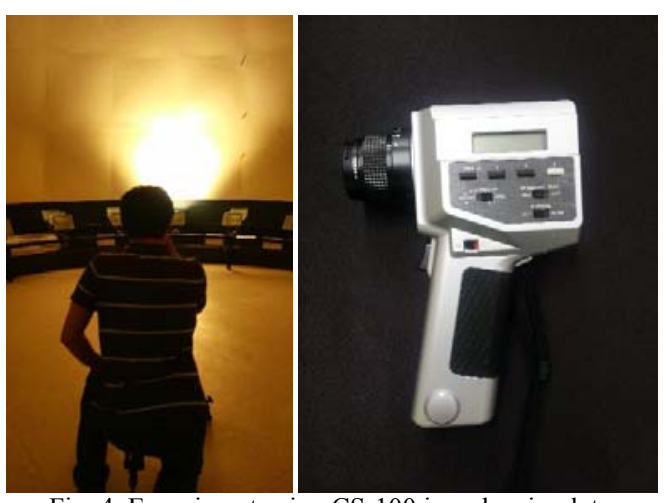

Fig. 4. Experiment using CS-100 in a sky simulator.

Fig. 4 shows the process of performing the experiment in the sky simulator. The matt material was attached on the target surface to reduce the effect of unintended reflection caused by room surfaces, which in turn could have a significant effect on the amount of the luminance measured. Also, in order to reduce the possibility of errors, the CS-100 luminance meter device was firmlyanchored on a tripod to limit movements or shaking

\section{RESULTS}

\section{A. Luminance Measurement in an Outdoor Environment} Depending on the Distance

This study focused on the difference displayed by measured luminance obtained through two different methods. The first measurements were obtained through the use of point luminance meter and the second measurements were obtained through the application of HDRI processing technique. Fig 5 shows the results obtained from outdoor lighting sources that were placed 4 meters away to 10 meters away in an identical measuring condition at night. The luminance values produced by HDRI processing was higher than those produced by CS-100 by about 1.5 times regardless of the distance. Also, the longer the measuring distance was, the smaller the luminance became. Thus, different algorithms are able to affect the luminance depending on the measuring distance.

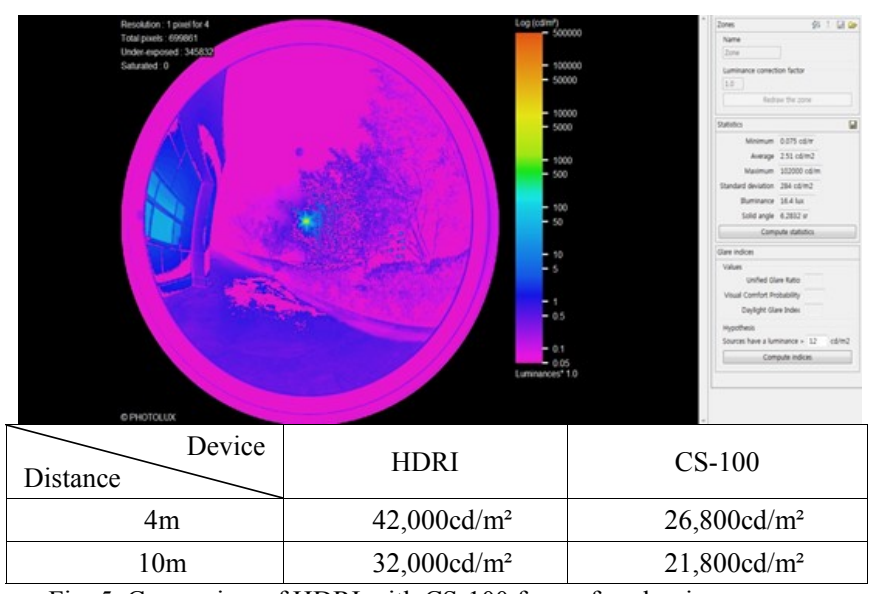

Fig. 5. Comparisonof HDRI with CS-100 for surface luminance.

\section{B. Luminance Interference by a Neighboring Light Source}

Since there may be great differences upon a direct measurement of the light source, the experiment was conducted at the measurement target surface, which is the same as the one used for other experiments. As explained in previous sections of this paper, this experiment was conducted inside a sky simulator in which two light sources were installed. One light source was considered as our target light source and the other one as the neighboring light source. During this study, we measured the luminance on the target area when the neighboring light source was switched off, switched and tilted to a 15 degree angle, and switched on and tilted at a 30 degree angle facing the luminance meter. The equipment used here was a CS-100 luminance meter positioned facing the front. Fig 6 shows the graph comparing the luminance values when the coherent light source is switched off, switched on and tilted to a 15 degree angle, and switched on and tilted to a 30 degree respectively from left to right.

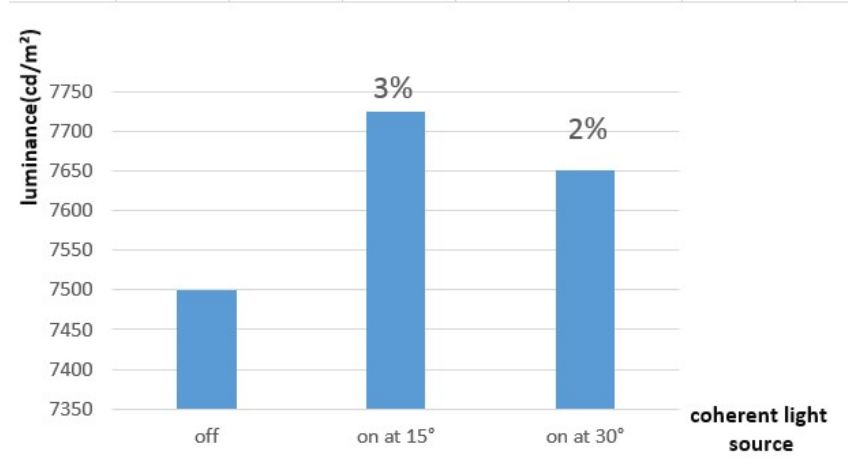

Fig. 6. Luminance with coherent lighting source.

\section{CONCLUSION}

This study intended to examine the changes in luminance caused by alterations in the distance between the point being 
targeted and the point at which measurements are carried out, measurement algorithms as well as to investigate the impact in terms of measured luminance that a neighboring source has on the target light source.

As the results show, the luminance changed depending on which measurement device was being used and the distance from which the target light source was away from the measuring point.The results also showed that the difference in luminance values under the same conditions was around $50 \%$ when the point and surface luminance meters were used. And that the distance away from the target light source, at which the measurements are taken has a significant effect on the measured luminance displayed by the target light source.

According to the experiment, on the impact of coherent light sources on measured luminance values, the luminance value was shown to be around 3\% higher when the coherent light source was switched on and oriented at a 15 degree angle from the front view and around $2 \%$ higher when it was switched on and oriented at a 30 degree angle from the front view.

The results obtained from this study imply that luminance values vary according to distance and that when a coherent light source exists near the measurement point, it can have a significant effect on the luminance values obtained when the target point is measured. Thus, the concepts provided by this study should be given a reasonable consideration when dealing with luminance measurements for the assessment of light pollution caused by diverse light sources and light fixtures or for the preparation of the "Act on Prevention of Light Pollution caused by Artificial Lighting".

\section{REFERENCES}

[1] C. Julio, Introduction to Nonimaging OptiCS, $1^{\text {st }}$ ed., Optical Science and Engineering, CRC Press, 2008, pp. 449.

[2] Y. Li, L. Sharan, and E. H. Adelson, "Compressing and companding high dynamic range images with subband architectures," ACM Trans. Graphics, vol. 24, no. 3, pp. 836-844, July 2005.

[3] H. Cai, "Camera aided luminance measurement of the luminous surfaces of different light sources, " in Proc. the AEI 2011 Conference AEI 2011. Building Integrated Solutions, pp. 272-279.

[4] J. Tumblin and H. Rushmeier, "Tone reproduction for realistic images," IEEE trans. Computer Graphics and Applications, vol. 13, no. 6, pp. 42-48, Nov. 1993.

[5] J. J. McCann and R. Alessandro, The Art and Science of HDR Imaging, John Wiley \& Sons press, vol. 26, 2011

[6] J. Axel, "High dynamic range imaging and its application in building research," Advances in Building Energy Research, vol. 1, no. 1, pp. 177-202, Sep. 2007.

[7] F. W. Campbell and D. G. Green, "Optical and retinal factors affecting visual resolution," The Journal of Physiology, vol. 181, no. 3, pp. 576, 1965.

[8] F. W. Campbell and R. W. Gubisch, "Optical quality of the human eye," The Journal of Physiology, vol. 186, no. 3, pp. 558-578, June, 1966.
[9] C. A. Johnson, "Effects of luminance and stimulus distance on accommodation and visual resolution," JOSA, vol. 66, no. 2, pp. 138-142, Feb. 1976

[10] E. Tural and T. Mehmedalp. Luminance Contrast Analyses for Low Vision in a Senior Living Facility: A Proposal for an HDR Image-Based Analysis Tool. [Online]. Available: http://www. ac.els-cdn.com/S0360132314001917/1-s2.0-S0360132314001917-ma in.pdf?_tid=1dc0a354-fc2a-11e3-a537-00000aab0f26\&acdnat=14036 74537 1bde 7b75dafc086497c4c69e4095322d.

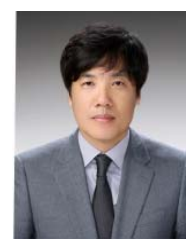

Gon Kim is a professor of Kyung Hee University in Republic of Korea. He got hisdoctoral degree from Texas A\&M University in the area of sustainable design for all-glazedatrium buildings. His professional life is densely filled with research, practice, publication, and teaching for the growth of environment-friendly architecture with sustainable lighting. His skill in computational analysis of building performance has been to provide the pragmatic knowledge available on how to design, develop, evaluate, construct and operate the healthiest, most productive and energy efficient built environment in Korea. He has presented lots of research outcomes on various aspects of the health design work in terms of visual environment. In his teaching role within the Department of Architectural Engineering at Kyung Hee University, he teaches core courses within his areas of interest, sustainable lighting, day lighting, energy efficiency and health-centric design, and more advanced methodologies to monitor the already-built environment.

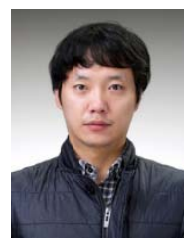

Hongsoo Lim is currently a Ph.D. student at the Department of Architectural Engineering in Kyung Hee University, Republic of South Korea. He has studied and published papers on low energy consumption of architectural environments and the usage of shading devices. In order to find methods and issues related to building energy consumption, he learned sustainable architecture theories while conducting 3 projects supported by the Korean government. His work is mainly centered on researching on new and trending methods of sustainable architecture as well as developing the already existing methods to better suit the current increasing need for low energy and sustainable buildings. He has outstanding skills in daylighting and building energy evaluation simulation programs such as RADIANCE, IES V.E and eQUEST for good visual environment and low energy buildings. He has also worked with the R\&D center of Hyundai Aluminum Corporation as an associate research engineer.

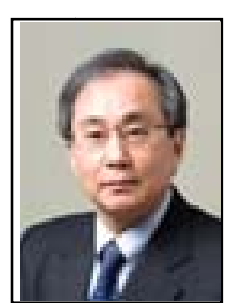

Jeong Tai Kim is a Kyung Hee Distinguished Fellow at Kyung Hee University and is a fellow of the Korea Academy of Science and Technology (KAST). In September of 2008, He founded the center for sustainable healthy buildings $(\mathrm{CSHeB})$ with the award of engineering research center (ERC) from Korea Government. As the director of the $\mathrm{CSHeB}$, he has tried to establish an outstanding R\&D center with global competitiveness by providing on-site research facilities, formulating synergistic interconnection with interdisciplinary researchers, and promoting collaboration and cooperation with various industries, government departments, and international universities. He also plays a key role in promoting sustainable healthy building technologies academically by hosting international symposiums, seminars, and workshops. He acted as the president of the Acoustical Society of Korea (2005), the Korea Institute of Ecological Architecture and Environment (2007-2008), and currently acts as the President of the Korea Society of Lighting and Visual Environment and the vice-president of the International Society of the Built Environment 